\title{
PREDICTIVE CONTROL OF TIME-DELAY PROCESSES
}

\author{
Marek Kubalčík, Vladimír Bobál \\ Tomas Bata University in Zlín \\ Faculty of Applied Informatics \\ Nad Stráněmí 4511 \\ 76005 Zlín \\ Czech Republic \\ E-mail: kubalcikl@fai.utb.cz
}

\section{KEYWORDS}

Predictive control, Time-delay systems, Digital control, Higher order systems, Simulation,

\begin{abstract}
In technical practice often occur higher order processes when a design of an optimal controller leads to complicated control algorithms. One of possibilities of control of such processes is their approximation by lower-order model with time-delay (dead time). One of the possible approaches to control of dead-time processes is application of predictive control methods. The paper deals with design of an algorithm for predictive control of high-order processes which are approximated by second-order model of the process with time-delay.
\end{abstract}

\section{INTRODUCTION}

Some technological processes in industry are characterized by high-order dynamic behaviour or large time constants and time-delays. Time-delay in a process increases the difficulty of controlling it. However using the approximation of higher-order process by lower-order model with time-delay provides simplification of the control algorithms.

Let us consider a continuous-time dynamical linear SISO (single input $u(t)$ - single output $y(t)$ ) system with time-delay $T_{d}$. The transfer function of a pure transportation lag is $e^{-T_{d} s}$ where $s$ is a complex variable. Overall transfer function with time-delay is in the form

$$
G_{d}(s)=G(s) e^{-T_{d} s}
$$

where $G(s)$ is the transfer function without time-delay. Processes with time-delay are difficult to control using standard feedback controllers. One of the possible approaches to control processes with time delay is predictive control. The predictive control strategy includes a model of the process in the structure of the controller. The first time-delay compensation algorithm was proposed by (Smith 1957). This control algorithm known as the Smith Predictor (SP) contained a dynamic model of the time-delay process and it can be considered as the first model predictive algorithm.
Model Based Predictive Control (MBPC) or only Predictive Control is one of the control methods which have developed considerably over a few past years. Predictive control is essentially based on discrete or sampled models of processes. Computation of appropriate control algorithms is then realized namely in the discrete domain.

The term Model Predictive Control designates a class of control methods which have common particular attributes (Camacho and Bordons 2004, Mikleš and Fikar 2008).

- Mathematical model of a systems control is used for prediction of future control of a systems output.

- The input reference trajectory in the future is known.

- A computation of the future control sequence includes minimization of an appropriate objective function (usually quadratic one) with the future trajectories of control increments and control errors.

- Only the first element of the control sequence is applied and the whole procedure of the objective function minimization is repeated in the next sampling period.

The principle of MBPC is shown in Fig. 1, where $u(t)$ is the manipulated variable, $y(t)$ is the process output and $w(t)$ is the reference signal, $\mathrm{N}_{1}, \mathrm{~N}_{2}$ and $\mathrm{N}_{\mathrm{u}}$ are called minimum, maximum and control horizon. This principle is possible to define as follows:

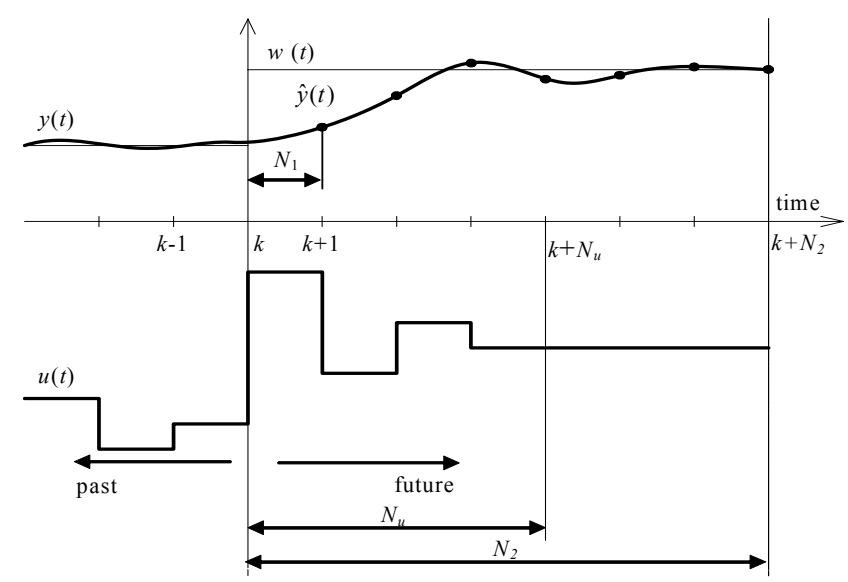

Figure 1: Principle of MBPC 
1. The process model is used to predict the future outputs $\hat{y}(t)$ over some horizon $N$. The predictions are calculated based on information up to time $k$ and on the future control actions that are to be determined.

2. The future control trajectory is calculated as a solution of an optimisation problem consisting of a objective function and possibility some constraints. The cist function comprises future output predictions, future reference trajectory, and future actions.

3. Although the whole future control trajectory was calculated in the previous step, only first element $u(k)$ is actually applied to the process. At the next sampling time the procedure is repeated. This is known as the Receding Horizon concept.

Theoretical research in the area of predictive control has a great impact on the industrial world and there are many applications of predictive control in industry. Its development has been significantly influenced by industrial practice. At present, predictive control with a number of real industrial applications belongs among the most often implemented modern industrial process control approaches. First predictive control algorithms were implemented in industry as an effective tool for control of multivariable industrial processes with constraints more than twenty five years ago. The use of predictive control was limited on control of namely rather slow processes due to the amount of computation required. At present, with the computing power available today, this is not an essential problem. A fairly actual and extensive surveys of industrial applications of predictive control are presented in (Morari and Lee 2004, Quin and Bandgwell 1996, 2000, 2003).

The aim of the paper is to design and verify by simulation an algorithm for predictive control of second order linear systems with time delay of two steps. A number of higher order industrial processes can be approximated by this model.

\section{IMPLEMENTATION OF PREDICTIVE CONTROL}

In this Section, GPC will be briefly described. The GPC method is in principle applicable to both SISO and MIMO processes and is based on input-output models. The standard cost function used in GPC contains quadratic terms of (possible filtered) control error and control increments on a finite horizon into the future

$$
J=\sum_{i=N_{1}}^{N_{2}} \delta(i)[\hat{y}(k+i)-w(k+i)]^{2}+\sum_{i=1}^{N_{u}}[\lambda(i) \Delta u(k+i-\mathbf{1})]^{2}
$$

where $\hat{y}(k+i)$ is the process output of $i$ steps in the future predicted on the base of information available upon the time $k, w(k+1)$ is the sequence of the reference signal and $\Delta u(k+i-1)$ is the sequence of the future control increments that have to be calculated. Implicit constraints on $\Delta u$ are placed between $N_{u}$ and $N_{2}$ as

$$
\Delta u(k+i-1)=0, \quad N_{u}<i \leq N_{2}
$$

The parameters $\delta(i)$ and $\lambda(i)$ are sequences which affect future behaviour of the controlled process. Generally, they are chosen in the form of constants or exponential weights.

\section{Calculation of the Optimal Control}

The objective of predictive control is a computation of a sequence of future increments of the manipulated variable $[\Delta u(k), \Delta u(k+1), \ldots]$ so that the criterion (2) was minimized. For further computation, it is necessary to transform the criterion (2) to a matrix form.

The output of the model (predictor) is computed as the sum of the free response $\boldsymbol{y}_{0}$ and the forced response of the model $\boldsymbol{y}_{n}$

$$
\hat{\boldsymbol{y}}=\boldsymbol{y}_{n}+\boldsymbol{y}_{0}
$$

It is possible to compute the forced response as the multiplication of the matrix $\boldsymbol{G}$ (Jacobian of the model) and the vector of future control increments $\Delta \boldsymbol{u}$, which is generally a priori unknown

$$
\boldsymbol{y}_{n}=\boldsymbol{G} \Delta \boldsymbol{u}
$$

where

$$
\boldsymbol{G}=\left[\begin{array}{ccccc}
g_{1} & 0 & 0 & \cdots & 0 \\
g_{2} & g_{1} & 0 & \cdots & 0 \\
g_{3} & g_{2} & g_{1} & \cdots & 0 \\
\vdots & \vdots & \vdots & \ddots & \vdots \\
g_{N_{2}} & g_{N_{2}-1} & g_{N_{2}-2} & \cdots & g_{N_{2}-N_{u}+1}
\end{array}\right]
$$

is matrix containing values of the step sequence.

It follows from equations (4) and (5) that the predictor in a vector form is given by

$$
\hat{\boldsymbol{y}}=\boldsymbol{G} \Delta \boldsymbol{u}+\boldsymbol{y}_{0}
$$

and the cost function (2) can be modified to the form below

$$
\begin{aligned}
J & =(\hat{\boldsymbol{y}}-\boldsymbol{w})^{T}(\hat{\boldsymbol{y}}-\boldsymbol{w})+\lambda \Delta \boldsymbol{u}^{T} \Delta \boldsymbol{u}= \\
& =\left(\boldsymbol{G} \Delta \boldsymbol{u}+\boldsymbol{y}_{0}-\boldsymbol{w}\right)^{T}\left(\boldsymbol{G} \Delta \boldsymbol{u}+\boldsymbol{y}_{0}-\boldsymbol{w}\right)+\lambda \Delta \boldsymbol{u}^{T} \Delta \boldsymbol{u}
\end{aligned}
$$

where $\boldsymbol{w}$ is the vector of future reference trajectory. Minimisation of the cost function (8) now becomes a direct problem of linear algebra. The solution in an unconstrained case can be found by setting partial derivative of $J$ with respect to $\Delta \boldsymbol{u}$ to zero and yields 


$$
\Delta \boldsymbol{u}=-\left(\boldsymbol{G}^{T} \boldsymbol{G}+\lambda \boldsymbol{I}\right)^{-1} \boldsymbol{G}^{T}\left(\boldsymbol{y}_{0}-\boldsymbol{w}\right)
$$

where the gradient $\boldsymbol{g}$ and Hessian $\boldsymbol{H}$ are defined as

$$
\begin{gathered}
\boldsymbol{g}^{T}=\boldsymbol{G}^{T}\left(\boldsymbol{y}_{0}-\boldsymbol{w}\right) \\
\boldsymbol{H}=\boldsymbol{G}^{T} \boldsymbol{G}+\lambda \boldsymbol{I}
\end{gathered}
$$

Equation (9) gives the whole trajectory of the future control increments and such is an open-loop strategy. To close the loop, only the first element $\boldsymbol{u}$, e. g. $\Delta u(k)$ is applied to the system and the whole algorithm is recomputed at time $k+1$. This strategy is called the Receding Horizon Principle and is one of the key issues in the MBPC concept.

If we denote the first row of the matrix $\left(\boldsymbol{G}^{T} \boldsymbol{G}+\lambda \boldsymbol{I}\right)^{-1} \boldsymbol{G}^{T}$ by $\boldsymbol{K}$ then the actual control increment can be calculated as

$$
\Delta u(k)=\boldsymbol{K}\left(\boldsymbol{w}-\boldsymbol{y}_{0}\right)
$$

\section{COMPUTATION OF PREDICTOR}

An important task is computation of predictions for arbitrary prediction and control horizons. Dynamics of most of processes requires horizons of length where it is not possible to compute predictions in a simple straightforward way. Recursive expressions for computation of the free response and the matrix $\boldsymbol{G}$ in each sampling period had to be derived. There are several different ways of deriving the prediction equations for transfer function models. Some papers make use of Diophantine equations to form the prediction equations (e.g. (Kwon et. al. 1992)). In (Rossiter, 2003) matrix methods are used to compute predictions. We derived a method for recursive computation of both the free response and the matrix of the dynamics.

Computation of the predictor for the time-delay system can be obtained by modification of the predictor for the corresponding system without a time-delay. At first we will consider the second order system without timedelay and then we will modify the computation of predictions for the time-delay system.

\section{Second Order System without Time-Delay}

The model is described by the transfer function

$$
\begin{gathered}
G\left(z^{-1}\right)=\frac{b_{1} z^{-1}+b_{2} z^{-2}}{1+a_{1} z^{-1}+a_{2} z^{-2}}=\frac{B\left(z^{-1}\right)}{A\left(z^{-1}\right)} \\
A\left(z^{-1}\right)=1+a_{1} z^{-1}+a_{2} z^{-2} ; B\left(z^{-1}\right)=b_{1} z^{-1}+b_{2} z^{-2}
\end{gathered}
$$

The model can be also written in the form

$$
A\left(z^{-1}\right) y(k)=B\left(z^{-1}\right) u(k)
$$

A widely used model in general model predictive control is the CARIMA model which we can obtain from the nominal model (15) by adding a disturbance model

$$
A\left(z^{-1}\right) y(k)=B\left(z^{-1}\right) u(k)+\frac{C\left(z^{-1}\right)}{\Delta} n_{c}(k)
$$

where $n_{c}(k)$ is a non-measurable random disturbance that is assumed to have zero mean value and constant covariance and the operator delta is $1-z^{-1}$. Inverted delta is then an integrator.

The polynomial $C\left(z^{-1}\right)$ will be further considered as $C\left(z^{-1}\right)=1$. The CARIMA description of the system is then in the form

$$
\Delta A\left(z^{-1}\right) y(k)=B\left(z^{-1}\right) \Delta u(k-1)+n_{c}(k)
$$

The difference equation of the CARIMA model without the unknown term $n_{c}(k)$ can be expressed as:

$$
\begin{aligned}
& y(k)=\left(1-a_{1}\right) y(k-1)+\left(a_{1}-a_{2}\right) y(k-2)+a_{2} y(k-3)+ \\
& +b_{1} \Delta u(k-1)+b_{2} \Delta u(k-2)
\end{aligned}
$$

It was necessary to compute three step ahead predictions in straightforward way by establishing of lower predictions to higher predictions. The model order defines that computation of one step ahead prediction is based on three past values of the system output. The three step ahead predictions are as follows

$$
\begin{aligned}
& \hat{y}(k+1)=\left(1-a_{1}\right) y(k)+\left(a_{1}-a_{2}\right) y(k-1)+a_{2} y(k-2)+ \\
& +b_{1} \Delta u(k)+b_{2} \Delta u(k-1) \\
& \hat{y}(k+2)=\left(1-a_{1}\right) y(k+1)+\left(a_{1}-a_{2}\right) y(k)+a_{2} y(k-1)+ \\
& +b_{1} \Delta u(k+1)+b_{2} \Delta u(k) \\
& \hat{y}(k+3)=\left(1-a_{1}\right) y(k+2)+\left(a_{1}-a_{2}\right) y(k+1)+a_{2} y(k)+ \\
& +b_{1} \Delta u(k+2)+b_{2} \Delta u(k+1)
\end{aligned}
$$

The predictions after modification can be written in a matrix form

$$
\begin{aligned}
& {\left[\begin{array}{l}
\hat{y}(k+1) \\
\hat{y}(k+2) \\
\hat{y}(k+3)
\end{array}\right]=\left[\begin{array}{ll}
g_{1} & 0 \\
g_{2} & g_{1} \\
g_{3} & g_{2}
\end{array}\right]\left[\begin{array}{l}
\Delta u(k) \\
\Delta u(k+1)
\end{array}\right]+\left[\begin{array}{llll}
p_{11} & p_{12} & p_{13} & p_{14} \\
p_{21} & p_{22} & p_{23} & p_{24} \\
p_{31} & p_{32} & p_{33} & p_{34}
\end{array}\right]\left[\begin{array}{l}
y(k) \\
y(k-1) \\
y(k-2) \\
\Delta u(k-1)
\end{array}\right]=} \\
& =\left[\begin{array}{cc}
b_{1} & 0 \\
b_{1}\left(1-a_{1}\right)+b_{2} & b_{1} \\
\left(a_{1}-a_{2}\right) b_{1}+\left(1-a_{1}\right)^{2} b_{1}+\left(1-a_{1}\right) b_{2} & b_{1}\left(1-a_{1}\right)+b_{2}
\end{array}\right]\left[\begin{array}{l}
\Delta u(k) \\
\Delta u(k+1)
\end{array}\right]+ \\
& +\left[\begin{array}{cc}
\left(1-a_{1}\right) & \left(a_{1}-a_{2}\right) \\
\left(1-a_{1}\right)^{2}+\left(a_{1}-a_{2}\right) & \left(1-a_{1}\right)\left(a_{1}-a_{2}\right)+a_{2}
\end{array}\right. \\
& \left(1-a_{1}\right)^{3}+2\left(1-a_{1}\right)\left(a_{1}-a_{2}\right)+a_{2} \quad\left(1-a_{1}\right)^{2}\left(a_{1}-a_{2}\right)+a_{2}\left(1-a_{1}\right)+\left(a_{1}-a_{2}\right)^{2} \\
& \left.\begin{array}{cc}
a_{2} & b_{2} \\
a_{2}\left(1-a_{1}\right) & b_{2}\left(1-a_{1}\right) \\
a_{2}\left(1-a_{1}\right)^{2}+\left(a_{1}-a_{2}\right) a_{2} & b_{2}\left(1-a_{1}\right)^{2}+\left(a_{1}-a_{2}\right) b_{2}
\end{array}\right]\left[\begin{array}{l}
y(k) \\
y(k-1) \\
y(k-2) \\
\Delta u(k-1)
\end{array}\right]
\end{aligned}
$$


It is possible to divide computation of the predictions to recursion of the free response and recursion of the matrix of the dynamics. Based on the three previous predictions it is repeatedly computed the next row of the free response matrix in the following way:

$$
\begin{aligned}
& p_{41}=\left(1-a_{1}\right) p_{31}+\left(a_{1}-a_{2}\right) p_{21}+a_{2} p_{11} \\
& p_{42}=\left(1-a_{1}\right) p_{32}+\left(a_{1}-a_{2}\right) p_{22}+a_{2} p_{12} \\
& p_{43}=\left(1-a_{1}\right) p_{33}+\left(a_{1}-a_{2}\right) p_{23}+a_{2} p_{13} \\
& p_{44}=\left(1-a_{1}\right) p_{34}+\left(a_{1}-a_{2}\right) p_{24}+a_{2} p_{14}
\end{aligned}
$$

The first row of the matrix is omitted in the next step and further prediction is computed based on the three last rows including the one computed in the previous step. This procedure is cyclically repeated. It is possible to compute an arbitrary number of rows of the matrix.

The recursion of the dynamics matrix is similar. The next element of the first column is repeatedly computed in the same way as in the previous case and the remaining columns are shifted to form a lower triangular matrix in the way which is obvious from the equation (16). This procedure is performed repeatedly until the prediction horizon is achieved. If the control horizon is lower than the prediction horizon a number of columns in the matrix is reduced. Computation of the new element is performed as follows:

$$
g_{4}=\left(1-a_{1}\right) g_{3}+\left(a_{1}-a_{2}\right) g_{2}+a_{2} g_{1}
$$

\section{Second Order System with Time-Delay}

The nominal model with two steps time-delay is considered as

$$
G\left(z^{-1}\right)=\frac{B\left(z^{-1}\right)}{A\left(z^{-1}\right)} z^{-2}=\frac{b_{1} z^{-1}+b_{2} z^{-2}}{1+a_{1} z^{-1}+a_{2} z^{-2}} z^{-2}
$$

The CARIMA model for time-delay system takes the form

$$
\Delta A\left(z^{-1}\right) y(k)=z^{-d} B\left(z^{-1}\right) \Delta u(k-1)+n_{c}(k)
$$

where $d$ is the dead time. In our case $d$ is equal to 2 . In order to compute the control action it is necessary to determine the predictions from $d+1(2+1$ in our case) to $d+N_{2}\left(2+N_{2}\right)$.

The predictor (20) is then modified to

$$
\begin{aligned}
& {\left[\begin{array}{l}
\hat{y}(k+3) \\
\hat{y}(k+4) \\
\hat{y}(k+5)
\end{array}\right]=\left[\begin{array}{lll}
p_{31} & p_{32} & p_{33} \\
p_{41} & p_{42} & p_{43} \\
p_{51} & p_{52} & p_{53}
\end{array}\right]\left[\begin{array}{c}
y(k) \\
y(k-1) \\
y(k-2)
\end{array}\right]+} \\
& +\left[\begin{array}{ll}
g_{1} & 0 \\
g_{2} & g_{1} \\
g_{3} & g_{2}
\end{array}\right]\left[\begin{array}{c}
\Delta u(k) \\
\Delta u(k+1)
\end{array}\right]+ \\
& +\left[\begin{array}{lll}
g_{2} & g_{3} & p_{34} \\
g_{3} & g_{4} & p_{44} \\
g_{4} & g_{5} & p_{54}
\end{array}\right]\left[\begin{array}{c}
\Delta u(k-1) \\
\Delta u(k-2) \\
\Delta u(k-3)
\end{array}\right]
\end{aligned}
$$

Recursive computation of the matrices is analogical to the recursive computation described in the previous section.

\section{SIMULATION EXAMPLES}

As simulation examples were chosen a fifth order linear system described by following transfer function

$$
G_{A}(s)=\frac{2}{(s+1)^{5}}=\frac{2}{s^{5}+5 s^{4}+10 s^{3}+10 s^{2}+5 s+1}
$$

and a fifth-order linear system with non-minimum phase

$$
G_{B}(s)=\frac{2(1-5 s)}{s^{5}+5 s^{4}+10 s^{3}+10 s^{2}+5 s+1}
$$

The systems were identified by the model (23) using off-line LSM (Bobál et. al., 2012). The system (26) was approximated by

$$
G_{A}\left(z^{-1}\right)=\frac{0,0424 z^{-1}+0,0296 z^{-2}}{1-1,6836 z^{-1}+0,7199 z^{-2}} z^{-2}
$$

and the system (27) was approximated by

$$
G_{B}\left(z^{-1}\right)=\frac{-0,7723 z^{-1}+0,8514 z^{-2}}{1-1,6521 z^{-1}+0,6920 z^{-2}} z^{-2}
$$

Both for sampling period $T_{0}=0.5 \mathrm{~s}$. The step responses of the models are in the following figures

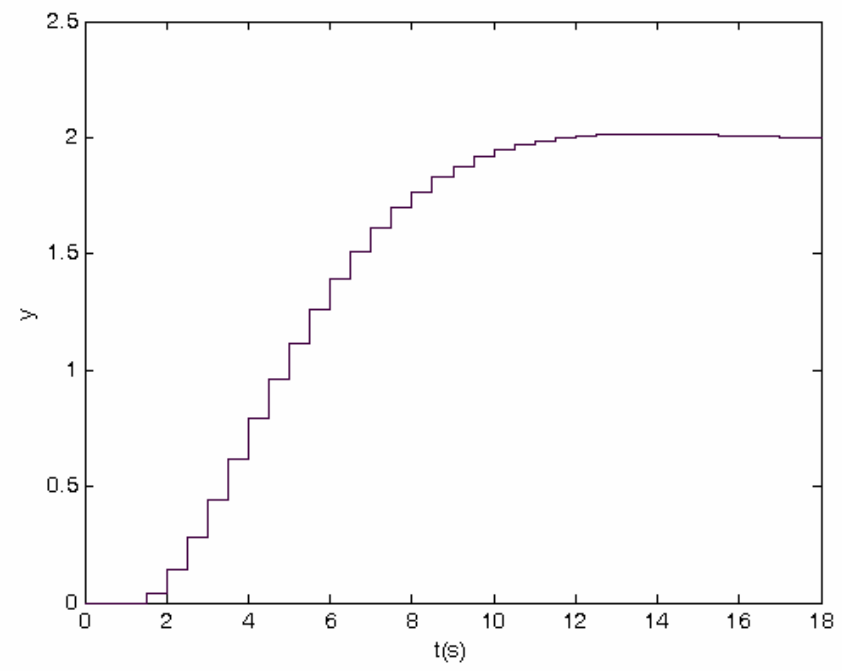

Figure 2: Step response of the model (28) 


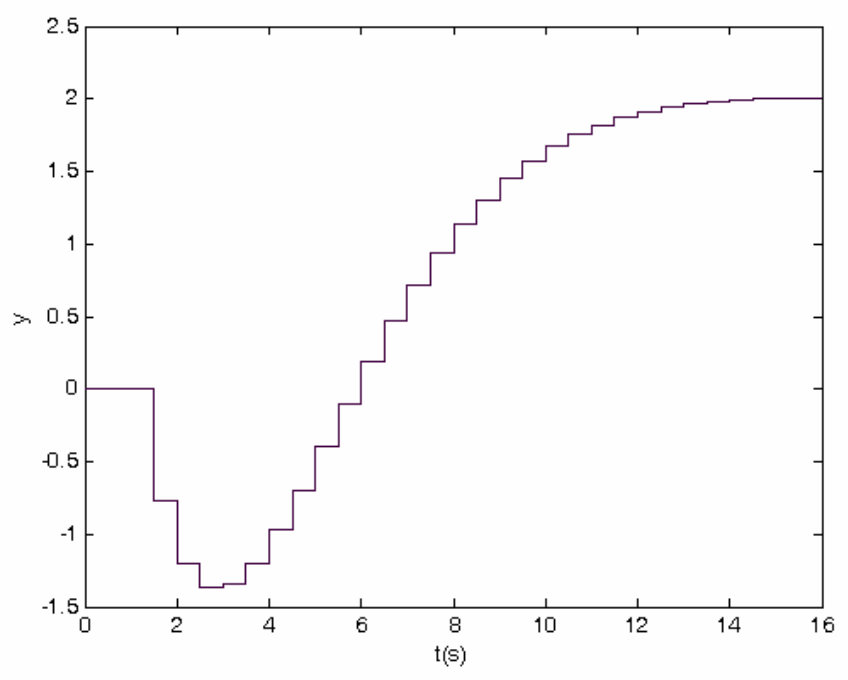

Figure 3: Step response of the model (29)

Control responses are in the figures 4, 5, 6 and 7.

The tuning parameters that are lengths of the prediction and control horizons and the weighting coefficient $\lambda$ were tuned experimentally. There is a lack of clear theory relating to the closed loop behavior to design parameters. The length of the prediction horizon, which should cover the important part of the step response, was in both cases set to $N=40$. The length of the control horizon was also set to $N_{u}=40$. The coefficient $\lambda$ was taken as equal to 0,5 .

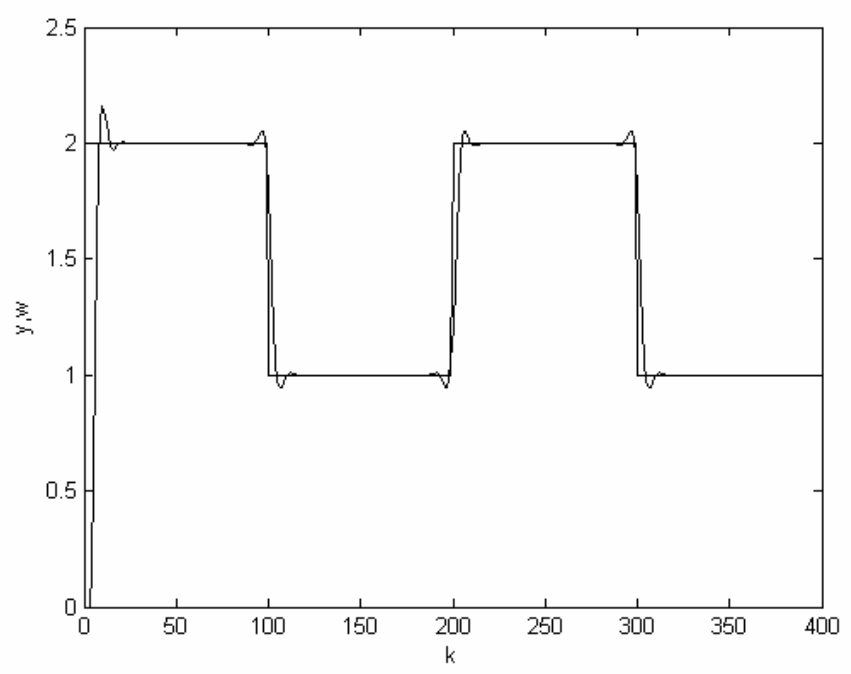

Figure 4: Control of the model (29)

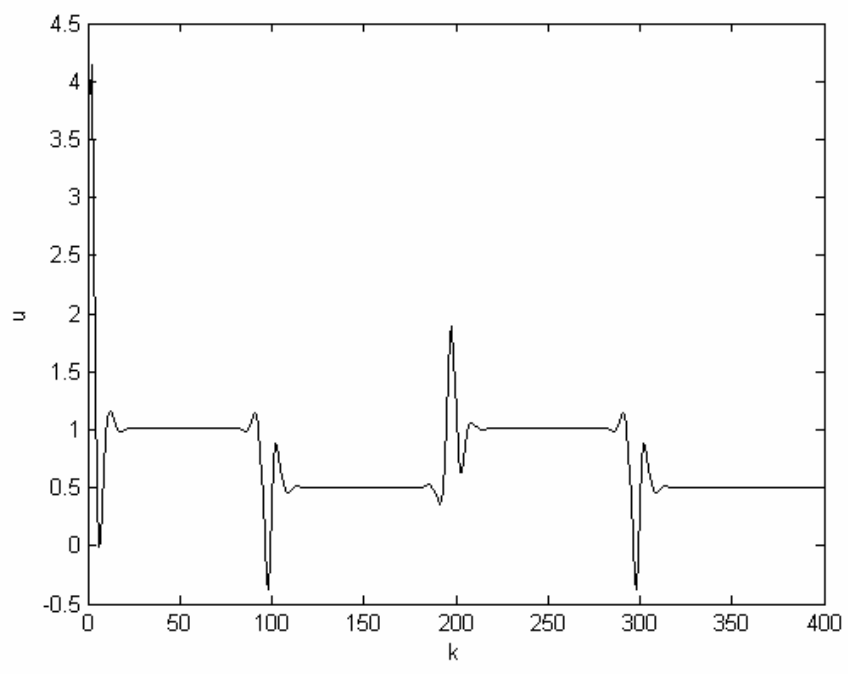

Figure 5: Control of the model (29) -manipulated variable

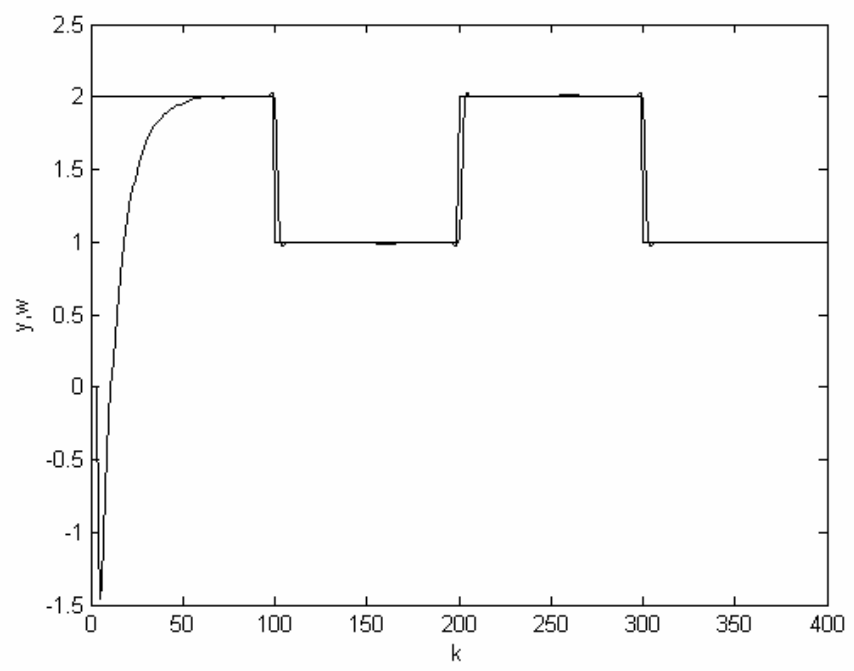

Figure 6: Control of the model (28)

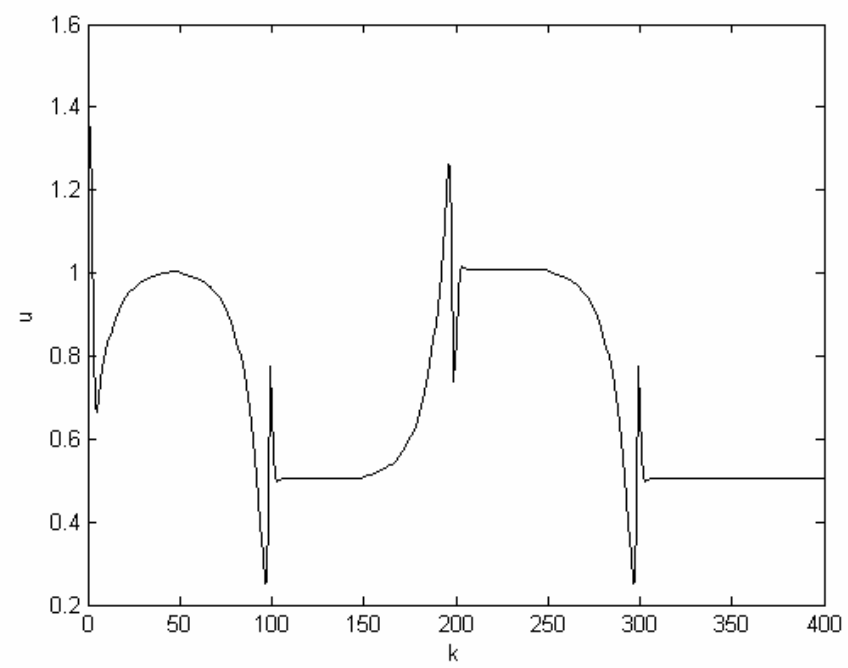

Figure 5: Control of the model (28) -manipulated variable 


\section{CONCLUSION}

The algorithm for control of the higher-order processes based on model predictive control was designed. The higher-order process was approximated by the secondorder model with time delay. The predictive controller is based on the recursive computation of predictions by direct use of the CARIMA model. The computation of predictions was extended for the timedelay system. The control of two modifications of the higher-order processes (stable and non-minimum phase) were verified by simulation. The simulation verification provided good control results. Asymptotic tracking of the reference signal was achieved in both cases. The control of non-minimum phase system was rather sensitive to tuning parameters. Experimental tuning of the controller was more complicated in this case. The algorithm will be tested and verified by real-time control of a heat-exchanger.

\section{ACKNOWLEDGMENTS}

This article was created with support of Operational Programme Research and Development for Innovations co-funded by the European Regional Development Fund (ERDF), national budget of Czech Republic within the framework of the Centre of Polymer Systems project (reg. number: CZ.1.05/2.1.00/03.0111).

\section{REFERENCES}

Bobál, V., Kubalčík, M., Chalupa, P. and Dostál, P. 2012. "Identification and Digital Control of Highre-Order Processes Using Predictive Strategy". These proceedings.

Camacho E. F., Bordons, C. 2004. Model Predictive Control. Springer-Verlag, London.

Kwon, W. H., Choj, H., Byun, D.G., Noh, S. 1992. "Recursive solution of generalized predictive control and its equivalence to receding horizon tracking control.“ Automatica, 28(6), 1235-1238.

Mikleš, J., Fikar, M. 2008. Process Modelling, Optimisation and Control. Springer-Verlag, Berlín.

Morari, M., Lee, J.H. 1999. "Model predictive control: past, present and future." Computers and Chemical Engineering, 23(4), 667-682.

Quin, S.J, Bandgwell, T.A. 1996. "An overview of industrial model predictive control technology." Proceedings of the Chemical Process Control - V. Vol. 93 of AIChE Symposium Series. CACHE \& AIChE. Tahoe City, CA, USA, 232-256.

Quin, S.J, Bandgwell, T.A. 2000. "An overview of nonlinear model predictive control applications." Nonlinear Model Predictive Control (F. Allgöwer \& A. Zheng, Ed.), (Basel - Boston - Berlin: Birkhäuser Verlag), 369-392.

Quin, S.J, Bandgwell, T.A. 2003. "A survey of industrial model predictive control technology." Control Engineering Practice, 11(7), 733-764.

Rossiter, J.A. 2003. Model Based Predictive Control: a Practical Approach, CRC Press.

Smith, O.J. 1957. "Closed control of loops". Chem. Eng. Progress 53, 217-219.

\section{AUTHOR BIOGRAPHIES}

MAREK KUBALČÍK graduated in 1993 from the Brno University of Technology in Automation and Process Control. He received his Ph.D. degree in Technical Cybernetics at Brno University of Technology in 2000. From 1993 to 2007 he worked as senior lecturer at the Faculty of Technology, Brno University of Technology. From 2007 he has been working as an associate professor at the Department of Process Control, Faculty of Applied Informatics of the Tomas Bata University in Zlín, Czech Republic. Current work cover following areas: control of multivariable systems, selftuning controllers, predictive control. His e-mail address is: kubalcik@fai.utb.cz.. You can contact him on email address dostalp@fai.utb.cz

VLADIMÍR BOBÁL graduated in 1966 from the Brno University of Technology, Czech Republic. He received his Ph.D. degree in Technical Cybernetics at Institute of Technical Cybernetics, Slovak Academy of Sciences, Bratislava, Slovak Republic. He is now Professor at the Department of Process Control, Faculty of Applied Informatics of the Tomas Bata University in Zlín. His research interests are adaptive control and predictive control, system identification and CAD for automatic control systems. You can contact him on email address bobal@fai.utb.cz. 